\title{
ON PSEUDOPOINTS OF ALGEBRAIC CURVES
}

\author{
REZA R. FARASHAHI AND IGOR E. SHPARLINSKI
}

\begin{abstract}
Following Kraitchik and Lehmer, we say that a positive integer $n \equiv 1(\bmod 8)$ is an $x$-pseudosquare if it is a quadratic residue for each odd prime $p \leq x$, yet is not a square. We extend this defintion to algebraic curves and say that $n$ is an $x$-pseudopoint of a curve $f(u, v)=0$ (where $f \in \mathbb{Z}[U, V]$ ) if for all sufficiently large primes $p \leq x$ the congruence $f(n, m) \equiv 0(\bmod p)$ is satisfied for some $m$.

We use the Bombieri bound of exponential sums along a curve to estimate the smallest $x$-pseudopoint, which shows the limitations of the modular approach to searching for points on curves.
\end{abstract}

\section{INTRODUCTION}

Following Lehmer in [9], given a real $x \geq 1$, we say that a nonsquare positive integer $n$ is an $x$-pseudosquare if $n \equiv 1(\bmod 8)$ and $(n / p)=1$ for each odd prime $p \leq x$, see also [13, 15, 16, 17] for further results. Here we generalise this notion and introduce and study $x$-pseudopoints on algebraic curves.

More precisely, given an absolutely irreducible polynomial $f(U, V) \in$ $\mathbb{Z}[U, V]$ and an integer $q \geq 1$ we denote

$$
\mathcal{Z}_{f}(q)=\{(n, m): 0 \leq n, m<q, f(n, m) \equiv 0 \quad(\bmod q)\} .
$$

Then, we define $\mathcal{P}_{f}$ as the set of primes $p$ for which $\mathcal{Z}_{f}(p)$ is not empty. We note that an absolutely irreducible polynomial $f$ remains absolutely irreducible modulo all sufficiently large prime numbers $p$ by Ostrowski [11]. Therefore, by the Weil bound, see [10, Section VIII.5, Bound (5.7)], we conclude that $\mathcal{P}_{f}$ contains all sufficiently large primes. In particular, for

$$
M_{f}(x)=\prod_{p \in \mathcal{P}_{f}(x)} p
$$

by the prime number theorem, we have

$$
M_{f}(x)=\exp ((1+o(1)) x) .
$$

Date: November 13, 2018. 
Furthermore for a real $x \geq 1$, we denote by $\mathcal{P}_{f}(x)=\mathcal{P}_{f} \cap[2, x]$ and say that an integer $n \geq 0$ is an $x$-pseudopoint of $f$ if for all $p \in \mathcal{P}_{f}(x)$ we have $(n, m) \in \mathcal{Z}_{f}(p)$ for some $m$, but the equation $f(n, m)=0$ has no integer solution $m \in \mathbb{Z}$. We note that Bernstein [2] has introduced and studied this notion in the case of the polynomials of the form $f(U, V)=g(U)-V^{2}$.

Clearly, apart of the congruence condition $n \equiv 1(\bmod 8)$ and the coprimality condition $\operatorname{gcd}(n, p)=1$ for primes $p \leq x$, the polynomial $f(U, V)=U-V^{2}$ corresponds to the case of $x$-pseudosquares.

It is easy to show that for any absolutely irreducible polynomial $f(U, V) \in \mathbb{Z}[U, V]$ nonlinear in $V$, that is, $\operatorname{deg}_{V} f \geq 2$, the smallest $x$-pseudopoint $N_{f}(x)$ satisfies the bound

$$
N_{f}(x)=O\left(M_{f}(x)\right)=\exp ((1+o(1)) x) .
$$

Clearly the condition of nonlinearity in $V$ is necessary (for example, the polynomial $f(U, V)=U^{2}-V$ does not have any pseudopoints).

Indeed, the bound (3) can be derived from the Chinese remaindering theorem combined with the Weil bound (see [10, Section VIII.5, Bound (5.7)]), and also the bound of Bombieri and Pila [12] on the number of integer points on plane curves.

Here we use the Bombieri bound [3] to improve (3).

Theorem 1. For any absolutely irreducible polynomial

$$
f(U, V) \in \mathbb{Z}[U, V]
$$

that is nonlinear in $V$, that is,

$$
\operatorname{deg}_{V} f \geq 2
$$

we have

$$
N_{f}(x) \leq M_{f}(x)^{1 / 2+o(1)} .
$$

The bound of Theorem 1 is an analogues of similar, albeit stronger, estimates for pseudosquares, see [13, 15]. Besides it shows the limitations of the modular approach to searching for points on curves. Indeed, assuming that $\mathcal{P}_{f}$ consists of all primes (otherwise the equation $f(n, m)=0$ has no integer solutions), we see that there is a reasonably small point which is a solution to the corresponding congruence modulo all small primes but is not a solution to the equation.

\section{Preparations}

We need some background on exponential sums and congruences.

For an integer $q$ and a complex $z$, we denote

$$
\mathbf{e}_{q}(z)=\exp (2 \pi i z / q)
$$


and recall the identity

$$
\frac{1}{q} \sum_{-q / 2<a \leq q / 2} \mathbf{e}_{q}(\text { an })=\left\{\begin{array}{lll}
1, & \text { if } n \equiv 0 \quad(\bmod q), \\
0, & \text { if } n \neq 0 \quad(\bmod q),
\end{array}\right.
$$

which follows from the formula for the sum of geometric progression.

We also need the following bound

$$
\sum_{n=1}^{N} \mathbf{e}_{q}(a n)=O(\min \{N, q /|a|\}),
$$

which holds for any integers $a$ and $N \geq 1$ with $0<|a| \leq q / 2$, see [7, Bound (8.6)].

Our main tool is the following special case of the Bombieri bound [3, Theorem 6] of exponential sums along a curve.

Lemma 2. Assume that for a prime p, a polynomial $f(U, V) \in \mathbb{Z}[U, V]$ is such that its reduction modulo $p$ does not have a factor of the form $U-\alpha$ with some $\alpha \in \mathbb{F}_{p}$. Then uniformly over $a \in \mathbb{Z}$ with $\operatorname{gcd}(a, p)=1$

$$
\sum_{(u, v) \in \mathcal{Z}_{f}(p)} \mathbf{e}_{p}(a u)=O\left(p^{1 / 2}\right),
$$

where the implied constant depends only on $\operatorname{deg} f$.

Finally, we need the following consequence of the Chinese remainder theorem (see also [7, Equation (12.21)] for a very similar statement).

Lemma 3. For any polynomial

$$
f(U, V) \in \mathbb{Z}[U, V]
$$

we have

$$
\sum_{(u, v) \in \mathcal{Z}_{f}\left(M_{f}(x)\right)} \mathbf{e}_{M_{f}(x)}(a u)=\prod_{p \in \mathcal{P}_{f}(x)} \sum_{(u, v) \in \mathcal{Z}_{f}(p)} \mathbf{e}_{p}(a u) .
$$

Proof. Let $\mathbb{Z}_{m}$ denote the residue ring modulo $m$. From the Chinese remainder theorem, there is a bijection

$$
\mathbb{Z}_{M_{f}(x)} \simeq \bigotimes_{p \in \mathcal{P}_{f}(x)} \mathbb{Z}_{p}
$$

by $u \mapsto\left(u_{p}\right)_{p \in \mathcal{P}_{f}(x)}$. On the other hand, every tuple $\left(u_{p}\right)_{p \in \mathcal{P}_{f}(x)}$ corresponded to the unique element

$$
u=\sum_{p \in \mathcal{P}_{f}(x)} \frac{M_{f}(x)}{p} u_{p} \in \mathbb{Z}_{M_{f}(x)} .
$$


Then,

$$
\mathbf{e}_{M_{f}(x)}(a u)=\prod_{p \in \mathcal{P}_{f}(x)} \mathbf{e}_{p}\left(a u_{p}\right)
$$

Moreover, in a natural way (6) yields a bijection between the points $(u, v)$ in $\mathcal{Z}_{f}\left(M_{f}(x)\right)$ and the tuples of points

$$
\left(\left(u_{p}, v_{p}\right)\right)_{p \in \mathcal{P}_{f}(x)} \in \bigotimes_{p \in \mathcal{P}_{f}(x)} \mathcal{Z}_{f}(p)
$$

Therefore,

$$
\begin{aligned}
\sum_{(u, v) \in \mathcal{Z}_{f}\left(M_{f}(x)\right)} \mathbf{e}_{M_{f}(x)}(a u) & =\sum_{\substack{\left(\left(u_{p}, v_{p}\right)\right)_{p \in \mathcal{P}_{f}(x)} \\
\left(u_{p}, v_{p}\right) \in \mathcal{Z}_{f}(p)}} \prod_{p \in \mathcal{P}_{f}(x)} \mathbf{e}_{p}\left(a u_{p}\right) \\
& =\prod_{p \in \mathcal{P}_{f}(x)} \sum_{(u, v) \in \mathcal{Z}_{f}(p)} \mathbf{e}_{p}(a u)
\end{aligned}
$$

which completes the proof.

Lemma 4. For any absolutely irreducible polynomial

$$
f(U, V) \in \mathbb{Z}[U, V]
$$

there is a constant $c>0$, depending only on $f$ such that

$$
\prod_{p \in \mathcal{P}_{f}(x)}\left(p+c p^{1 / 2}\right) \geq \# \mathcal{Z}_{f}\left(M_{f}(x)\right) \geq \prod_{p \in \mathcal{P}_{f}(x)} \max \left\{1, p-c p^{1 / 2}\right\} .
$$

Proof. By the Chinese remaindering theorem we have a bijection between points of $\mathcal{Z}_{f}\left(M_{f}(x)\right)$ and tuples of points in $\prod_{p \in \mathcal{P}_{f}(x)} \mathcal{Z}_{f}(p)$, see the proof of Lemma 3. Therefore,

$$
\# \mathcal{Z}_{f}\left(M_{f}(x)\right)=\prod_{p \in \mathcal{P}_{f}(x)} \# \mathcal{Z}_{f}(p)
$$

As we have noted, the polynomial $f$ remains absolutely irreducible modulo all sufficiently large prime numbers $p$ by Ostrowski [11]. Let $c_{f}$ be the least integer such that $f$ is absolutely irreducible over $\mathbb{Z}_{p}$ for all prime numbers $p \geq c_{f}$ (for explicit bounds on $c_{f}$ see for example [6, 14, 18]). If $f$ is absolutely irreducible over $\mathbb{Z}_{p}$, and $p \geq c_{f}$ then from the Weil bound we have

$$
\# \mathcal{Z}_{f}(p)=p+O\left(p^{1 / 2}\right)
$$

see [10, Section VIII.5, Bound (5.7)]. Furthermore, allowing the implied constant in (7) to depend of $f$, we see that (7) trivially holds for all primes $p$. By definition, for all $p \in \mathcal{P}_{f}(x)$ we have $\# \mathcal{Z}_{f}(p) \geq 1$, which completes the proof. 
Corollary 5. For any absolutely irreducible polynomial

$$
f(U, V) \in \mathbb{Z}[U, V]
$$

we have

$$
\# \mathcal{Z}_{f}\left(M_{f}(x)\right)=M_{f}(x)^{1+o(1)}
$$

Proof. Since

$$
\begin{aligned}
p \exp \left(c_{0} p^{-1 / 2}\right) & \geq p+c p^{1 / 2} \geq \max \left\{1, p-c p^{1 / 2}\right\} \\
& \geq p \exp \left(-c_{0} p^{-1 / 2}\right)
\end{aligned}
$$

for an appropriate constant $c_{0} \geq 0$, we have

$$
\begin{aligned}
\# \mathcal{Z}_{f}\left(M_{f}(x)\right) & =M_{f}(x) \exp \left(O\left(\sum_{p \leq x} p^{-1 / 2}\right)\right) \\
& =M_{f}(x) \exp \left(O\left(x^{1 / 2} / \log x\right)\right),
\end{aligned}
$$

which together with (2) implies the result

Finally we need the following estimate on the number of points on a curve with a restricted coordinate which follows from a result of Pila [12] that in turn slightly improves the previous estimate of Bombieri and Pila [4].

Lemma 6. For any absolutely irreducible polynomial

$$
f(U, V) \in \mathbb{Z}[U, V]
$$

nonlinear in $V$ that is,

$$
\operatorname{deg}_{V} f \geq 2
$$

the equation

$$
f(n, m)=0, \quad 0 \leq n<N, m \in \mathbb{Z},
$$

has at most $O\left(N^{1 / 2+o(1)}\right)$ solutions.

Proof. Let $d=\operatorname{deg} f$. Since $\operatorname{deg}_{V} f \geq 2$ we see that for any solution to the above equation we have $m=O\left(n^{d / 2}\right)=O\left(N^{d / 2}\right)$. Recalling that by [12], an absolutely irreducible polynomial of degree $d$ has $O\left(T^{1 / d+o(1)}\right)$ solutions in a box $[0, T] \times[0, T]$ (where the implied constants depend only on $d$ ), we derive the result. 


\section{Proof of Theorem 1}

Let $T_{f}(N ; x)$ be the number of solutions $(n, m)$ to the congruence

$$
f(n, m) \equiv 0 \quad\left(\bmod M_{f}(x)\right), \quad 0 \leq n<N, 0 \leq m<M_{f}(x) .
$$

Using (4), we write

$$
\begin{aligned}
T_{f}(N ; x) & =\sum_{(n, m) \in \mathcal{Z}_{f}\left(M_{f}(x)\right)} \sum_{k=0}^{N-1} \frac{1}{M_{f}(x)} \sum_{-M_{f}(x) / 2<a \leq M_{f}(x) / 2} \mathbf{e}_{M_{f}(x)}(a(n-k)) \\
& =\frac{1}{M_{f}(x)} \sum_{-M_{f}(x) / 2<a \leq M_{f}(x) / 2} \sum_{(n, m) \in \mathcal{Z}_{f}\left(M_{f}(x)\right)} \mathbf{e}_{M_{f}(x)}(a n) \sum_{k=0}^{N-1} \mathbf{e}_{M_{f}(x)}(-a k) .
\end{aligned}
$$

Separating the term corresponding to $a=0$, and recalling (5), we obtain

$$
T_{f}(N ; x)-\frac{N}{M_{f}(x)} \# \mathcal{Z}_{f}\left(M_{f}(x)\right)=O\left(R_{f}(N ; x)\right),
$$

where, as before, $\mathcal{Z}_{f}(q)$ is defined by (1) and

$$
R_{f}(N ; x)=\sum_{0<|a| \leq M_{f}(x) / 2} \frac{1}{|a|}\left|\sum_{(n, m) \in \mathcal{Z}_{f}\left(M_{f}(x)\right)} \mathbf{e}_{M_{f}(x)}(a n)\right| .
$$

To estimate $R_{f}(N ; x)$, for every $d \mid M_{f}(x)$, we collect together the values of $a$ with the same value $\operatorname{gcd}\left(a, M_{f}(x)\right)=d$ and write them as $a=d b$, getting

$$
R_{f}(N ; x)=\sum_{d \mid M_{f}(x)} \frac{1}{d} \sum_{\substack{0<|b| \leq M_{f}(x) / 2 d \\ \operatorname{gcd}\left(b, M_{f}(x) / d\right)=1}} \frac{1}{|b|}\left|\sum_{(n, m) \in \mathcal{Z}_{f}\left(M_{f}(x)\right)} \mathbf{e}_{M_{f}(x)}(d b n)\right| .
$$

Recalling Lemma 3 and then estimating the corresponding exponential sums via Lemma 2 for $p \nmid d$ (and using the trivial bound $\# \mathcal{Z}_{f}(p)$ for $p \mid d)$, we deduce $\left|\sum_{(n, m) \in \mathcal{Z}_{f}\left(M_{f}(x)\right)} \mathbf{e}_{M_{f}(x)}(d b n)\right| \leq C^{\pi(x)}\left(M_{f}(x) / d\right)^{1 / 2} d=C^{\pi(x)}\left(M_{f}(x) d\right)^{1 / 2}$, 
where $C$ is the implied constant of Lemma 2 and, as usual, $\pi(x)$ is the number of primes $p \leq x$. Therefore, using (2)

$$
\begin{aligned}
R_{f}(N ; x) & \leq C^{\pi(x)} \sum_{d \mid M_{f}(x)} \frac{1}{d} \sum_{\begin{array}{c}
0<|b| \leq M_{f}(x) / 2 d \\
\operatorname{gcd}\left(b, M_{f}(x) / d\right)=1
\end{array}} \frac{\left(M_{f}(x) d\right)^{1 / 2}}{|b|} \\
& =C^{\pi(x)} M_{f}(x)^{1 / 2} \sum_{d \mid M_{f}(x)} \frac{1}{d^{1 / 2}} \sum_{\substack{0<|b| \leq M_{f}(x) / 2 d \\
\operatorname{gcd}\left(b, M_{f}(x) / d\right)=1}} \frac{1}{|b|} \\
& =O\left((2 C)^{\pi(x)} M_{f}(x)^{1 / 2} \log M_{f}(x)\right)=O\left(M_{f}(x)^{1 / 2+o(1)}\right),
\end{aligned}
$$

as trivially

$$
\sum_{d \mid M_{f}(x)} \frac{1}{d^{1 / 2}} \leq \sum_{d \mid M_{f}(x)} 1 \leq 2^{\pi(x)} .
$$

Thus, we see from (9) that

$$
\left|T_{f}(N ; x)-\frac{N}{M_{f}(x)} \# \mathcal{Z}_{f}\left(M_{f}(x)\right)\right| \leq M_{f}(x)^{1 / 2+o(1)} .
$$

Furthermore, by Corollary 5 we see that for any fixed $\varepsilon>0$, taking $N=\left\lceil M_{f}(x)^{1 / 2+\varepsilon}\right\rceil$ we obtain

$$
T_{f}(N ; x)=N^{1+o(1)} .
$$

By the Chinese remaindering theorem we see that for every fixed $n$ there are no more that $d^{\pi(x)}$ solutions $m$ to the congruence $f(n, m) \equiv 0$ $\left(\bmod M_{f}(x)\right), 0 \leq m<M_{f}(x)$. Thus we have at least

$$
T_{f}(N ; x) d^{-\pi(x)}=T_{f}(N ; x) M_{f}(x)^{o(1)}=N^{1+o(1)} .
$$

values of $n$ for which the congruence $f(n, m) \equiv 0\left(\bmod M_{f}(x)\right)$, has a solution. Using Lemma 6 we see that there is a solution $(n, m)$ to (8) with $f(n, m) \neq 0$. Since $\varepsilon$ is arbitrary. Since $\varepsilon$ is arbitrary, this concludes the proof.

\section{Comments}

It is easy to see that all implicit constants in our estimates can be efficiently evaluated. For example, see [6, 14, 18] for explicit versions of the Ostrowski theorem.

We remark that besides pseudosquares, in a number of works [1, 15, 8] the notion of pseudopowers has been studied. Namely, following Bach, Lukes, Shallit and Williams [1, we say that an integer $n>0$ is an $x$-pseudopower to base $g$ (for a given integer $g$ with $|g| \geq 2$ ) if $n$ is not a power of $g$ over the integers but is a power of $g$ modulo all primes 
$p \leq x$, that is, if for all primes $p \leq x$ there exists an integer $k_{p} \geq 0$ such that $n \equiv g^{k_{p}}(\bmod p)$.

The notion of pseudopowers naturally extends to elliptic curves. More precisely, given an elliptic curve $E$ over $\mathbb{Q}$ and a rational point $P \in E(\mathbb{Q})$ we say that $Q$ is an $x$-pseudomultiple of $P$ if for every prime $p \leq x$ at which $E$ has good reduction, there is an integer $k_{p} \geq 0$ so that $Q \equiv k_{p} P(\bmod p)$ but $Q$ is not of the form $Q=k P+T$ for some integer $k$ and some torsion point $T$. Clearly $E$ has to be of rank at least 2 for $x$-pseudomultiple to exist. Obtaining upper bounds on the canonical height of the smallest pseudopowers is a natural and challenging questions.

\section{ACKNOWLEDGEMENTS.}

The authors are grateful to Joe Silverman for the idea of the question on $x$-pseudomultiples on elliptic curves in Section 4, in fact this also gave us the idea of defining and studying $x$-pseudopoints on curves.

Both authors were supported in part by ARC Grant DP0881473.

\section{REFERENCES}

[1] E. Bach, R. Lukes, J. Shallit and H. C. Williams, 'Results and estimates on pseudopowers', Math. Comp., 65 (1996), 1737-1747.

[2] D. J. Bernstein, 'Doubly focused enumeration of locally square polynomial values', High Primes and Misdemeanours: Lectures in Honour of the 60th Birthday of Hugh Cowie Williams, Fields Institute Communications, vol.41, Amer. Math. Soc., 2004, 69-76.

[3] E. Bombieri, 'On exponential sums in finite fields', Amer. J. Math., 88 (1966), 71-105.

[4] E. Bombieri and J. Pila, 'The number of integral points on arcs and ovals', Duke Math. J., 59 (1989), 337-357.

[5] J. Bourgain, S. Konyagin, C. Pomerance and I. E. Shparlinski, 'On the smallest pseudopower', Acta Arith., 140 (2009), 43-55.

[6] S. Gao and V. M. Rodrigues, 'Irreducibility of polynomials modulo $p$ via Newton polytopes', Journal of Number Theory, 101 (2003), 32-47.

[7] H. Iwaniec and E. Kowalski, Analytic number theory, Colloquium Pubs., Vol. 53, Amer. Math. Soc., Providence, RI, 2004.

[8] S. V. Konyagin, C. Pomerance, and I. E. Shparlinski, 'On the distribution of pseudopowers', Can. J. Math., (to appear).

[9] D. H. Lehmer, 'A sieve problem on "pseudo-squares"', Math. Tables and Other Aids to Computation, 8 (1954), 241-242.

[10] D. Lorenzini, An invitation to arithmetic geometry, Amer. Math. Soc., 1996.

[11] A. Ostrowski, 'Zur arithmetischen theorie der algebraischen grössen', Nachr. K. Ges. Wiss. Göttingen (1919), 273-298.

[12] J. Pila, 'Density of integer points on plane algebraic curves, Intern. Math. Research Notices, 18 (1996), 903-912. 
[13] C. Pomerance, and I. E. Shparlinski, 'On pseudosquares and pseudopowers', Combinatorial Number Theory, Proc. of Integers Conf. 2007, Walter de Gruyter, Berlin, 2009, 171-184.

[14] W. M. Ruppert, 'Reducibility of polynomials $f(x ; y)$ modulo $p$ ', J. Number Theory, $\mathbf{7 7}$ (1999), 62-70.

[15] A. Schinzel, 'On pseudosquares', New trends in probability and statistics, $P a$ longa, 1996, Vol. 4, 213-220, VSP, Utrecht, 1997.

[16] J. P. Sorenson, 'The pseudosquares prime sieve', Proc. '7th Algorithmic Number Theory Symp., Lect. Notes in Comput. Sci., vol. 4076, Springer-Verlag, Berlin, 2006, 193-207.

[17] K. Wooding and H. C. Williams, 'Doubly-focused enumeration of pseudosquares and pseudocubes', Proc. 7th Algorithmic Number Theory Symp., Lect. Notes in Comput. Sci., vol. 4076, Springer-Verlag, Berlin, 2006, 208-211.

[18] U. Zannier, 'On the reduction modulo $\mathrm{p}$ of an absolutely irreducible polynomial $f(x ; y)$ ', Arch. Math., 68 (1997), 129-138.

Department of Computing, Faculty of Science, Macquarie UniverSity, Sydney, NSW 2109, Australia

E-mail address: $\{$ reza,igor\}@comp.mq.edu.au 